\title{
Intérêt de l'échographie dans la prise en charge du blessé de guerre dyspnéique
}

\author{
Use of Ultrasound for War Casualty Patient in an Austere Environment
}

\author{
P.-V. Martin · J. Fogelman $\cdot$ C. Dubecq $\cdot$ J. Galant $\cdot$ S. Travers $\cdot$ N. Fritsch \\ Reçu le 28 février 2018; accepté le 8 août 2018 \\ (C) SFMU et Lavoisier SAS 2018
}

Résumé La médecine militaire s'adapte aux dernières évolutions médicales, et l'échographie s'inscrit désormais dans les algorithmes de prise en charge des blessés de guerre. Grâce à la plus-value apportée à la clinique dans l'évaluation des blessés associée à l'amélioration des performances et à la miniaturisation des appareils, le positionnement de l'échographie en médecine de guerre a évolué pour trouver sa place " à l'avant ", parfois dans des conditions sanitaires très dégradées. Après avoir rappelé les principes du secourisme au combat pour le blessé de guerre dyspnéique, cet article replace l'intérêt diagnostique et thérapeutique de l'échographie dans la « médecine de l'avant » en détaillant la sémiologie échographique des principales pathologies du blessé de

\section{P.-V. Martin $(\bowtie)$}

Hôpital d'instruction des armées Percy,

1, rue du Lieutenant-Raoul-Batany,

F-92190 Clamart, France

e-mail : martin_paulvincent@yahoo.fr

\section{J. Fogelman}

Service d'accueil des urgences,

hôpital d'instruction des armées Percy,

1, rue du Lieutenant-Raoul-Batany,

F-92190 Clamart, France

C. Dubecq

Centre médical des armées Pau-Bayonne-Tarbes

Citadelle Général Bergé, BP 12,

F-64109 Bayonne Cedex, France

J. Galant

1re antenne médicale spécialisée

34, rue de la Martinière F-78000 Versailles-Satory, France

S. Travers

2e centre médical des armées, école du Val-de-Grâce,

BA 107, F-78129 Villacoublay, France

N. Fritsch

Service de réanimation,

hôpital d'instruction des armées Robert-Picqué,

351 Route de Toulouse,

F- 33882 Villenave d'Ornon Cedex, France guerre dans la phase de réanimation préhospitalière. L'article souligne aussi l'importance opérationnelle qu'occupe l'échographie, que ce soit sur le terrain lors des évacuations sanitaires ou lors d'afflux massif de victimes. Certains aspects de la doctrine militaire d'emploi pourraient s'adapter à la pratique en préhospitalier ou lors des situations d'exception en métropole.

Mots clés Échographie · Blessé de guerre · Réanimation préhospitalière

Abstract Military medicine follows medical evolutions in order to optimize the management of war-wounded patients. The ultrasound device is a powerful tool capable of providing additional information to the clinical examination. Its performance and miniaturization permit to transfer it at the battle field and to include it in the "forward medicalization". This article reminds principles of tactical combat casualty care for patient with a dyspnea and, explain the place that sonography can take to confirm a diagnosis. It explains the ultrasound semiology of main pathologies and underlines the operational impact of sonography during a large influx of injured patients and during the medical evacuation. This military practice can be adapted to prehospital care or to an uncommon situation like terrorism attack.

Keywords Ultrasonography · Combat casualty care · Remote damage control resuscitation

\section{Introduction}

La mission du Service de santé des armées (SSA) est d'assurer le soutien sanitaire des soldats. La doctrine française du « sauvetage au combat » a la particularité d'inclure une « médicalisation de l'avant » en plaçant une équipe sanitaire sur le terrain au plus près des hommes [1,2]. Cette médicalisation n'a cessé d'évoluer au cours de l'histoire pour 
intégrer les dernières avancées médicales et pour s'adapter aux nouvelles formes de conflits. L'échographie est l'un des progrès technologiques qui ont marqué la médecine d'urgence ces dernières années, jusque dans sa pratique préhospitalière. Maintenant que la miniaturisation des appareils en a fait un moyen diagnostique transportable, le médecin militaire a commencé à l'intégrer dans sa pratique sur le terrain.

En rappelant les bases du sauvetage au combat et tout en prenant en compte les contraintes imposées par le contexte opérationnel, cet article précise les indications et la place que peut prendre l'échographe dans la médicalisation de l'avant en se focalisant sur le blessé de guerre en détresse respiratoire.

\section{Échographie en médecine militaire}

Avant chaque départ sur un théâtre d'opérations extérieur, tous les personnels de santé (médecin, infirmier, auxiliaire sanitaire) bénéficient d'une préparation spécifique organisée par l'École du Val-de-Grâce via des structures de formation réparties sur le territoire métropolitain. Elles assurent l'enseignement de la doctrine du sauvetage au combat qui permet la mise en condition et la survie du blessé de guerre, de sa blessure initiale jusqu'à son arrivée à la structure médicochirurgicale où il pourra bénéficier éventuellement d'un damage control chirurgical avant d'être évacué vers la métropole [3]. La méthode enseignée d'évaluation clinique du blessé de guerre est standardisée et suit l'acronyme mnémotechnique « SAFE MARCHE RYAN » (Fig. 1).

Les médecins bénéficient également d'un stage d'apprentissage de l'échographie durant lequel sont appris l'eFAST (extended focused assessment with sonography for trauma) et des notions utiles pour gérer les urgences médicales en milieu isolé correspondant au premier niveau de compétence en échographie clinique $[4,5]$.

L'échographie, que ce soit pour un patient isolé ou dans le cas de situations multivictimes avec nécessité d'un triage médical, a su s'imposer dans la médicalisation de l'avant dans les derniers théâtres d'opérations où l'étendue géographique des zones de conflit augmente les délais d'évacuation. Les échographes sont positionnés dans les postes de secours mais également " à l'avant» dans les véhicules de transports terrestres et aériens. La miniaturisation des échographes permet même leur transport à dos d'homme. L'examen échographique est utilisé pour préciser le bilan lésionnel qui va guider la réanimation préhospitalière. Elle est décrite par les Anglo-Saxons sous le terme de prolonged field care [6]. Cette notion correspond au principe de remote damage controle resuscitation qui vise à débuter précocement et donc dès la phase préhospitalière l'ensemble des mesures réanimatoires pour le maintien en vie et la préservation du pronostic fonctionnel des blessés [7]. Ces mesures comprennent notamment le monitorage du patient, l'optimisation de

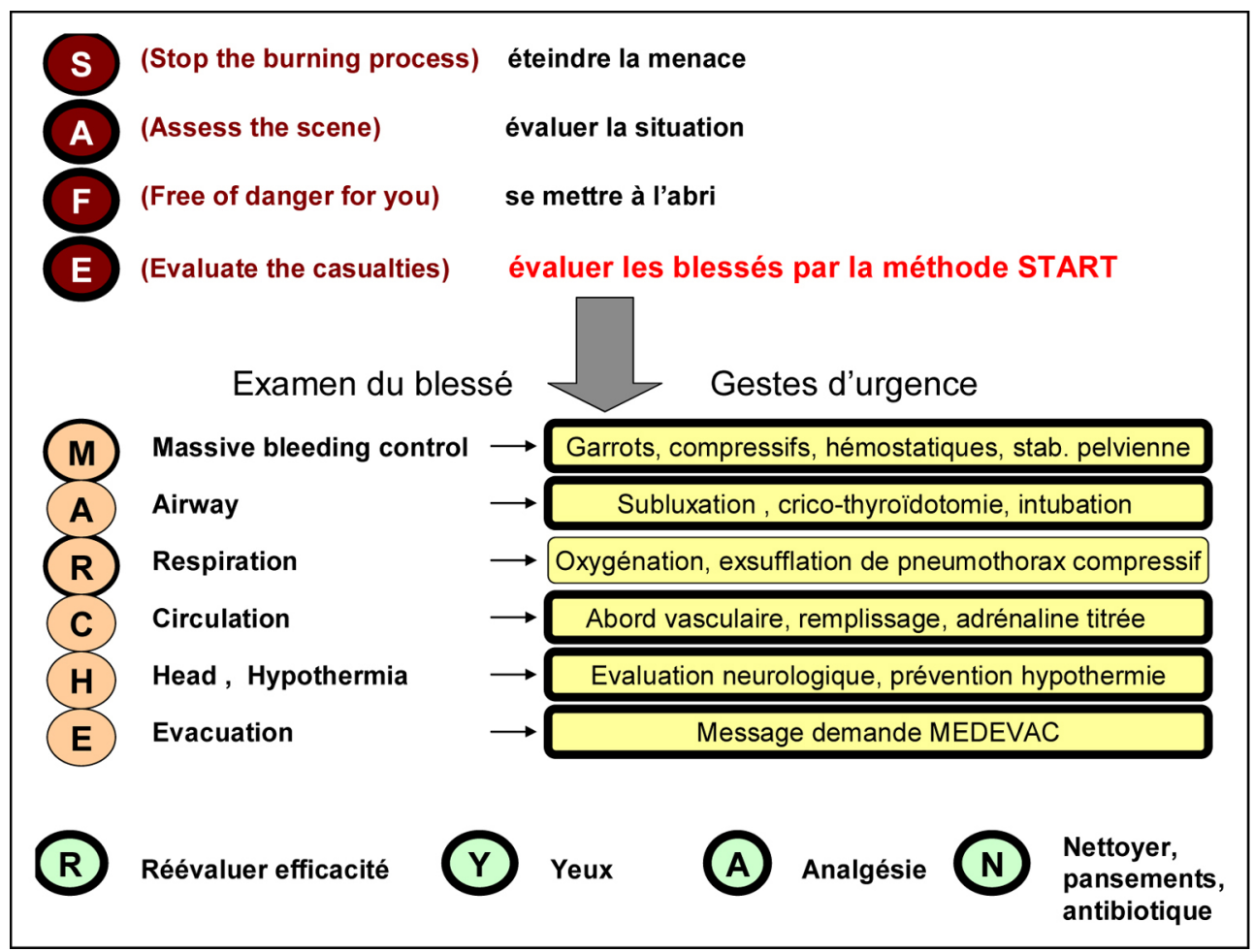

Fig. 1 SAFE MARCHE RYAN méthode pédagogique de la prise en charge des blessés de guerre par les équipes médicales françaises [2] 
l'analgésie, de la ventilation artificielle et de l'hémodynamique, ainsi que la gestion de la stratégie transfusionnelle par l'administration de produits sanguins labiles (concentrés globulaires, plasma cryodesséché et/ou sang frais total) [8]. L'échographie y trouve sa place et participe à la mise en condition du patient lors de son évacuation vers une structure adaptée pour une hémostase chirurgicale qui doit être la plus rapide possible, puisque le taux de survie est corrélé à la rapidité d'arrivée au bloc opératoire [9].

\section{Dyspnée du blessé de guerre}

Les détresses respiratoires des blessés de guerre sont souvent multifactorielles. L'analyse statistique des récents conflits montre que les soldats sont le plus souvent blessés par balle ou par les explosions des armes conventionnelles ou des IED (improvised explosive devices) [10]. Les patients atteints au thorax, au vu de la violence des mécanismes, doivent d'emblée être considérés comme des blessés graves puisque, selon les dernières recommandations sur les traumatisés thoraciques [9], une lésion pénétrante, un blast ou un traumatisme fermé du thorax causé par une forte énergie constituent des critères de gravité. Les blessures, qui seront décrites par la suite, peuvent s'associer et se potentialiser pour majorer une hypoxémie mettant rapidement en jeu le pronostic vital :

- les blessés peuvent présenter une obstruction des voies aériennes supérieures qui est responsable de $1 \%$ des morts au combat [10]. Elle se rencontre dans les comas, les traumatismes craniofaciaux ou cervicaux ou lors d'œdèmes de la face causés par des brûlures. Une prise en charge spécifique est nécessaire ;

- les traumatismes thoraciques sont présents chez un blessé sur dix. Les traumatismes pénétrants entraînent des lésions pleuropulmonaires qui exposent au risque de pneumothorax et d'hémothorax (40 et $17 \%$ respectivement dans la série de Keneally et Szpisjak [11]). Quatrevingts pour cent d'entre eux sont pris en charge par la pose d'un drain thoracique ou par une chirurgie thoracique dans la série de Lesquen et al. [12]. Le pneumothorax, lorsqu'il devient suffocant, peut rapidement devenir mortel et est responsable de $5 \%$ des décès au combat. Il est décrit comme une cause dite de «mort évitable », puisqu'il peut être traité par une exsufflation à l'aiguille. L'hémothorax massif constitue une entité clinique qui associe une dyspnée à un état de choc hémorragique. Les éléments de protection individuelle des soldats (gilets pareéclats) ont permis de limiter la survenue de ces traumatismes, mais ils restent fréquents chez les combattants qui n'en sont pas dotés. Il est important de rappeler que tout impact thoracique doit faire suspecter une atteinte des gros vaisseaux et du cœur; une tamponnade péricardique doit être évoquée en cas d'état de choc. De même, du fait de l'incertitude du trajet projectilaire, les plaies peuvent être thoracoabdominales, nécessitant en cas d'état de choc de rechercher un saignement intra-abdominal ;

- les lésions fermées du thorax sont également fréquentes [13]. Elles se rencontrent lors de lésions de blast ou lors de la projection des personnels dans l'habitacle de leur véhicule soumis à une explosion ou à un retournement. Les chocs directs entrainent des lésions pariétales comme des fractures de côtes ou des volets costaux rencontrés dans 25 et $3 \%$ des traumatismes thoraciques [11], ainsi que des contusions pulmonaires fréquentes (46\%) qui peuvent se compliquer secondairement d'une détresse respiratoire aiguë [14]. De façon plus anecdotique, les contusions pulmonaires se retrouvent aussi lorsqu'une balle est stoppée par un gilet de protection. L'énergie cinétique transmise entraine une contusion par " effet arrière » sans mécanisme pénétrant.

Parmi les causes de dyspnées au combat peut aussi être citée l'exposition à la toxicité des fumées, voire à des agents chimiques. Enfin, une tamponnade péricardique ou un état de choc hémorragique peuvent être la cause d'une dyspnée alors qu'il n'y a pas de lésion pulmonaire.

\section{Gestion de la détresse respiratoire en situation isolée}

Dans le contexte opérationnel, le sauvetage au combat repose avant tout sur l'examen clinique. Il est parfois rendu difficile du fait des conditions d'exercice, mais il est souvent suffisant pour déterminer l'étiologie d'une dyspnée et pour entreprendre les gestes de sauvetage adéquats. En cas de détresse respiratoire aiguë, deux extrêmes urgences sont à traiter :

- l'obstruction des voies aériennes supérieures : elle est la troisième cause de mort évitable sur le champ de bataille, et les soignants militaires sont deux fois plus exposés à cette problématique que les civils [15]. Tout ce qui obstrue les voies aériennes doit être rapidement enlevé (sang, liquide digestif, corps étrangers...). En cas d'échec ou de récidive de cette obstruction avec apparition d'une asphyxie, une cricothyroïdotomie doit être réalisée pour permettre l'oxygénation du patient. Également appelée coniotomie percutanée, elle se réalise grâce à un dispositif dédié (Mini-Track ${ }^{\circledR}$, Smiths Medical, Minneapolis, États-Unis) qui est introduit dans la trachée après incision au niveau de la membrane intercricothyroïdienne (MICT). Elle se situe entre le cartilage thyroïdien situé au-dessus et le cartilage cricoïde en dessous qui constituent les deux repères accessibles à la palpation pour déterminer la zone de ponction. La réalisation d'une cricothyroïdotomie chirurgicale avec une sonde 
d'intubation trachéale de petit calibre peut s'envisager en cas de nécessité de protection des voies aériennes ou de ventilation artificielle [16]. En cas de difficulté de localisation de la MICT (obésité, hématome du cou, peau brûlée) ou d'échec (18\% des cas dans la série de Barnard et al. [17]), un échorepérage pourrait aider à la localiser [18] ;

- le pneumothorax suffocant : il met rapidement en jeu le pronostic vital. Le diagnostic est avant tout clinique : le patient blessé déshabillé présente une détresse respiratoire aiguë avec une polypnée et une respiration superficielle. Le thorax a une ampliation asymétrique avec un hémichamp quasi immobile sous tension, amenant à un arrêt respiratoire. Les veines jugulaires sont turgescentes sauf chez le patient exsangue. L'hémodynamique est altérée, avec une hypotension artérielle qui est cliniquement évaluée par la perte du pouls radial (PAS inférieure à $80 \mathrm{mmHg}$ ) et qui peut se compliquer d'un arrêt circulatoire.

Face à un pneumothorax suffocant, tout doit être mis en œuvre pour aborder la plèvre afin de la décomprimer. Il est conseillé en première intention une exsufflation avec un cathéter de $14 \mathrm{G}$ d'au moins $5 \mathrm{~cm}$ au niveau du deuxième espace intercostal en médioclaviculaire. En cas d'échec, une thoracostomie au doigt ou à la pince doit être réalisée. L'échographie peut trouver un intérêt majeur pour confirmer le diagnostic parfois rendu difficile par l'agitation du patient et l'environnement dégradé ou éviter une décompression inutile. Lorsque le diagnostic clinique est non équivoque, le geste salvateur doit rester la priorité.

\section{Mise en condition de survie du blessé thoracique}

Le blessé conscient est mis en position demi-assise (en l'absence d'état de choc) avec de l'oxygène (si elle est disponible) et est instaurée sans tarder une analgésie efficace (morphine, kétamine...). Le patient inconscient doit être mis en position latérale de sécurité du côté de l'atteinte pulmonaire suspectée pour faciliter la mécanique ventilatoire.

La plaie soufflante et le volet costal sont deux lésions thoraciques qui doivent être prises en charge de façon spécifique devant leur risque de décompensation. Non traitées, les plaies soufflantes peuvent favoriser la survenue d'un pneumothorax suffocant. Pour l'éviter, un dispositif tel qu'une valve Asher$\operatorname{man}^{\circledR}$ ou la confection d'un pansement trois côtés doit être positionné sur ce type de plaies pour permettre l'échappement d'air tout en empêchant sa réentrée qui peut rendre le pneumothorax compressif. Les plaies soufflantes ne doivent jamais être obturées sauf en cas d'hémorragie abondante, et dans ce cas, un drainage pleural est réalisé de manière concomitante en évitant d'utiliser l'orifice de la plaie.
Le volet costal se repère cliniquement par la palpation et visuellement par son déplacement inversé par rapport aux mouvements respiratoires. Il se définit par au moins trois côtes successives fracturées à deux endroits différents sur leur trajet. Un bandage thoracique facilite la mécanique ventilatoire et évite un épuisement du patient. Une analgésie multimodale doit toujours y être associée pour limiter la douleur de chaque inspiration.

\section{Évaluation échographique du blessé}

L'évaluation échographique du blessé trouve sa place en complément de l'examen clinique sous réserve que les conditions sécuritaires soient satisfaisantes. Le but est de réaliser un bilan lésionnel plus exhaustif que le simple examen clinique afin de repérer des lésions susceptibles de s'aggraver, de débuter précocement des traitements adaptés et de guider les décisions thérapeutiques [19]. Dans le contexte des détresses vitales, l'échographie permet de mettre en évidence neuf fois sur dix des images caricaturales « illustrant» la gravité de l'état clinique [9]. En cas de dyspnée, l'échographie peut compléter l'évaluation thoracique clinique puis apporter des renseignements sur l'état hémodynamique.

\section{Examen échographique du thorax}

L'échographie pleuropulmonaire, déjà utilisée en pratique courante, a une bonne performance diagnostique dans la dyspnée du fait que la quasi-totalité des pathologies qui entrainent une détresse respiratoire atteignent la surface thoracique $[20,21]$. En traumatologie, une analyse échographique du thorax commence d'abord par la recherche des causes des urgences vitales telles que le pneumothorax par l'examen de la plèvre antérieure et l'épanchement pleural, témoin d'un hémothorax, par l'analyse de la plèvre postérieure. Une analyse plus fine peut rechercher secondairement une contusion pulmonaire ou des fractures costales en regard des zones traumatisées.

\section{Sémiologie de l'échographie pleuropulmonaire}

\section{Poumon sain}

Le poumon sain se définit échographiquement par la présence d'un glissement pleural objectivé par de petits artéfacts qui bougent en fonction des mouvements respiratoires au niveau de la ligne pleurale hyperéchogène. En mode échographique temps-mouvement (ou TM), il prend un aspect en « bord de mer » (Fig. 2), avec un « pouls pulmonaire » qui correspond à la transmission des mouvements cardiaques et vasculaires à la périphérie du poumon. 


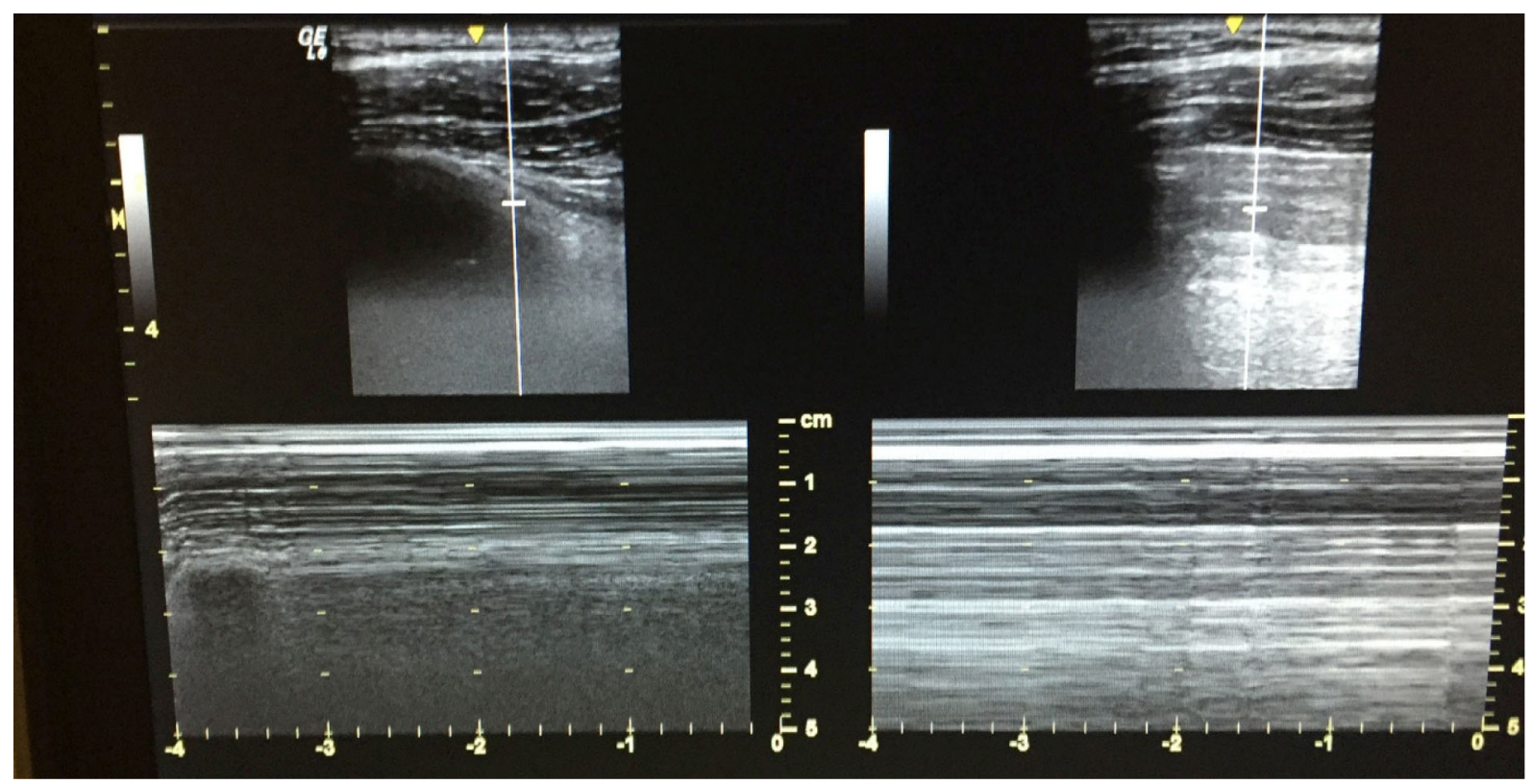

Fig. 2 Comparaison sur un même écran d'un poumon sain (à gauche) avec un aspect de « bord de mer » et d'un pneumothorax (à droite) avec un aspect de « code-barres » en $2 \mathrm{D}$ et en temps $\mathrm{M}$

\section{Pneumothorax}

Le diagnostic échographique de pneumothorax associe une absence de glissement pleural, un aspect en « code-barres » en mode TM, avec la perte du pouls pulmonaire (Fig. 2). Le « point-poumon » est un signe pathognomonique du pneumothorax qui marque l'endroit où le poumon se décolle de la paroi [20]. Si l'appareil le permet, une comparaison des deux poumons sur un même écran permet de bien identifier l'asymétrie des deux hémichamps (en l'absence de pneumothorax bilatéral) (Fig. 2).

La présence d'un glissement pleural élimine un pneumothorax de manière certaine à l'endroit où se situe la sonde. Il peut être absent lors de pathologies médicales comme lors d'un talcage, de la présence de larges bulles d'emphysème, d'obésité, de fibrose pulmonaire ou d'antécédent de chirurgie thoracique [22]. Un emphysème sous-cutané, rencontré dans les pneumothorax ou les atteintes des voies respiratoires, peut constituer une véritable barrière à l'interprétation des images.

L'usage de l'échographie pleurale en traumatologie est intéressant et a montré sa supériorité par rapport à la radiographie thoracique pour le diagnostic des épanchements intrathoraciques liquidiens ou gazeux au déchocage [21]. Une autre étude en traumatologie a montré que l'association d'une radio pulmonaire à l'admission et d'une échographie obtient une sensibilité de détection des pneumothorax à $97 \%$ en comparaison au scanner, les faux-négatifs étant des pneumothorax paracardiaques sans répercussion clinique immédiate [9].
Sur le terrain, l'attitude thérapeutique (surveillance rapprochée ou drainage thoracique) dépendra du contexte. En pratique préhospitalière civile, les indications de décompression thoracique sont limitées aux épanchements (pneumothorax et/ou hémothorax) mettant en jeu le pronostic vital immédiat [9]. Dans les conditions d'exception, avec des problèmes de surveillance possiblement rencontrés, une décision de drainage thoracique peut être prise pour éviter toute dégradation pendant la mise en attente en cas de victimes multiples ou d'évacuation sanitaire.

\section{Hémothorax}

Le diagnostic clinique de l'hémothorax est difficile surtout en conditions opérationnelles. En échographie, il se repère facilement sous la forme d'une zone déclive anéchogène intrathoracique dans laquelle le poumon surnage. Il s'agit d'un examen particulièrement performant (sensibilité de 84 à $97 \%$ et spécificité à $100 \%$ [9]). L'épaisseur de l'épanchement permet d'en évaluer la quantité : une taille maximale mesurée entre la paroi thoracique et le poumon supérieure à $50 \mathrm{~mm}$ en décubitus dorsal permet d'estimer un volume liquidien intrathoracique à plus de $800 \mathrm{ml}$ (Fig. 3) [22]. En médecine de guerre ou en situation isolée, cette quantification est importante. Un hémothorax volumineux dans un contexte de choc hémorragique constitue un argument fort pour réaliser un drainage thoracique avec un dispositif de recueil pour permettre une autotransfusion du sang récupéré, pour demander l'apport de culots globulaires avec 
le moyen d'évacuation et pour déclencher la procédure de collecte de sang total dans la structure d'accueil.

\section{Contusion pulmonaire}

Détectable à l'auscultation par des crépitants, l'échographie objective une contusion par la présence de grandes lignes B localisées au niveau des zones contuses (Fig. 4). Ces lignes B sont issues de la ligne pleurale, descendent sans épuisement sur tout l'écran tels des " faisceaux laser » en effaçant les lignes A horizontales physiologiques [20]. Leur présence évoque un syndrome interstitiel que l'on retrouve de façon bilatérale dans l'œdème aigu du poumon.

La contusion pulmonaire est la plus fréquente des blessures thoraciques sur le champ de bataille, mais est rarement responsable de façon isolée d'une détresse respiratoire, bien que quelques cas soient rapportés [11-14]. La dégradation de l'hématose survient lorsqu'elle est étendue ou du fait de l'installation progressive d'un œdème pulmonaire qui peut débuter dans les deux à six premières heures; elle peut alors évoluer de façon assez imprévisible vers un syndrome de détresse respiratoire aiguë (SDRA). En cas d'hypoxie et de contusion pulmonaire isolée, l'instauration d'une ventilation non invasive (VNI) peut constituer un traitement alternatif pour éviter une intubation [23]. En préhospitalier, l'échographie permet une utilisation sans arrière-pensée de la VNI après avoir éliminé sa contre-indication que constitue le pneumothorax.

\section{Bronchospasme}

Dans le bronchospasme, le poumon ne prend pas un aspect spécifique, mais ressemble échographiquement à un poumon
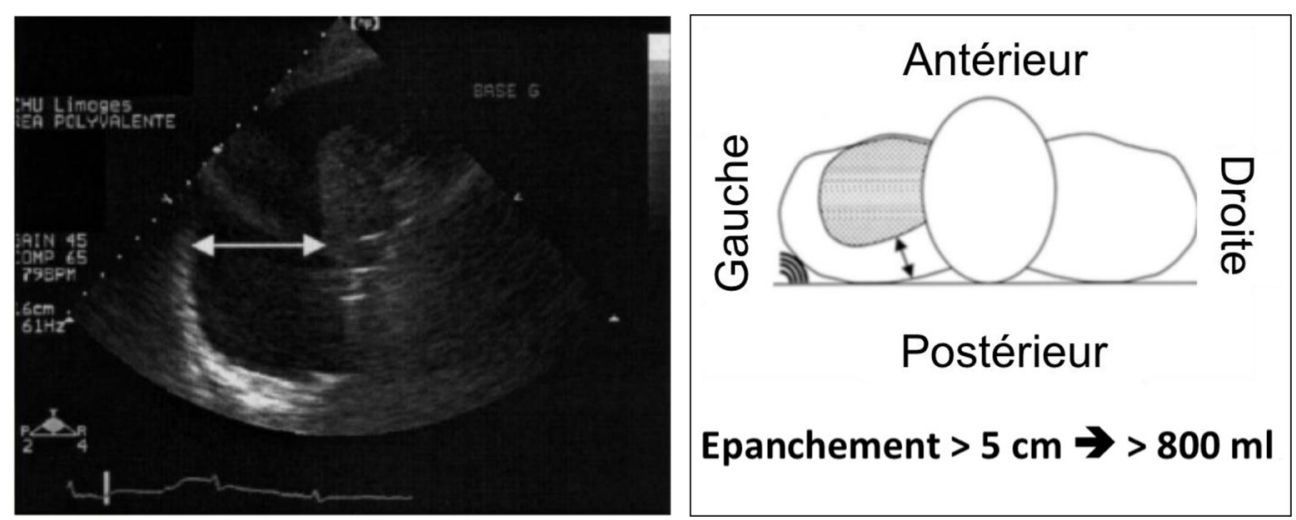

Fig. 3 La détection d'un hémothorax de plus de $5 \mathrm{~cm}$ entre la paroi et le poumon est quantifiée à au moins $800 \mathrm{ml}$ [22]
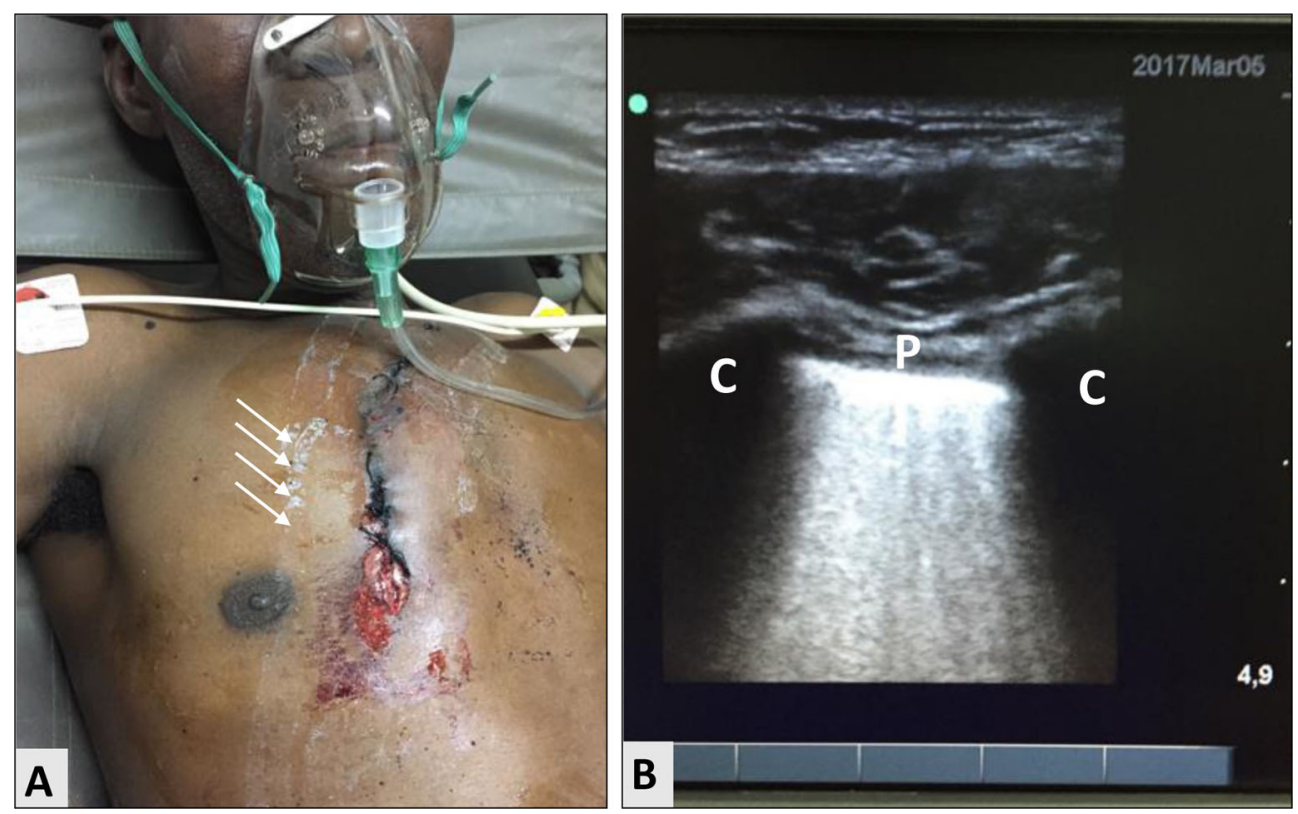

Fig. 4 A. Plaie par balle en séton sur le thorax droit (flèches : indication de l'emplacement de la sonde d'échographie pour l'obtention de l'image B). B. Aspect échographique de la contusion pulmonaire avec de nombreuses lignes B (P : plèvre ; C : côtes) 
sain. L'intérêt de l'échographie peut néanmoins être utile au cours des évacuations sanitaires avec une ambiance sonore saturée qui rend l'auscultation pulmonaire impossible. Le diagnostic de bronchospasme peut alors être évoqué lors d'une désaturation inexpliquée en vol avec un poumon échographiquement sain et dans un contexte évocateur (exposition à des fumées ou lors de pathologies médicales).

\section{Fractures costales, volet thoracique}

Ces diagnostics sont avant tout cliniques. Les ruptures de la corticale sont identifiables en échographie. La localisation précise des traits de fracture peut guider une anesthésie locale à but antalgique, bien que l'effet soit limité et transitoire.

\section{Causes extrapulmonaires responsables de dyspnée}

\section{État de choc hémorragique}

En cas de choc hémorragique et en l'absence de saignement extériorisé, l'échographie est un outil indispensable pour rechercher un saignement occulte. Les traumatismes pénétrants thoraciques sont parfois associés à des lésions abdominales ou cardiaques ; il est donc licite de rechercher un saignement par la réalisation d'un examen systématique des « $3 \mathrm{P}$ », c'est-à-dire des trois séreuses (plèvre, péritoine, péricarde). Une hémorragie non contrôlable en préhospitalier doit faire adapter les objectifs tensionnels suivant le principe de l'hypotension artérielle permissive : l'objectif est une PAS à $80 \mathrm{mmHg}$ en l'absence d'un traumatisme crânien grave [24]. La visualisation d'un épanchement constitue un critère de gravité qui doit motiver une évacuation sanitaire la plus précoce possible pour un geste d'hémostase [25]. Un examen échographique réalisé trop précocement peut être faussement rassurant et doit donc être répété.

\section{Tamponnade péricardique}

Tout traumatisme pénétrant du thorax doit faire rechercher un épanchement péricardique. Le diagnostic repose essentiellement sur l'échographie cardiaque transthoracique en analysant le péricarde en coupe sous-xyphoïdienne. Cet examen, qui a une excellente valeur diagnostique [5], met en évidence en cas de tamponnade un épanchement circonférentiel responsable d'abord d'une compression des cavités droites pouvant aller jusqu'au swinging heart [26]. Le collapsus de l'oreillette droite constitue un signe de prétamponnade. En cas d'épanchement circonférentiel compressif avec une instabilité hémodynamique, l'échographie permet de guider la péricardiocentèse de sauvetage qui consiste à ponctionner 50 à $200 \mathrm{cc}$ de liquide devant permettre une amélio- ration hémodynamique parfois spectaculaire en attendant l'intervention chirurgicale salvatrice.

Dans le cadre de l'évaluation du blessé de guerre, l'analyse échographique de la VCI par voie sous-costale peut constituer la pierre angulaire du diagnostic d'une hypotension artérielle lorsqu'elle est associée à une analyse du péricarde. Elle permet chez le traumatisé de faire le diagnostic étiologique du choc en distinguant le choc hypovolémique en lien avec une hémorragie du choc obstructif retrouvé dans la tamponnade péricardique ou le pneumothorax compressif. Un état hypovolémique se caractérise par une VCI de petite taille $(<10 \mathrm{~mm})$ avec une grande variabilité en fonction du cycle respiratoire. Collabée à l'inspiration, elle peut constituer un bon repère pour diagnostiquer une hypovolémie franche et commencer un remplissage [27]. En cas de choc hémorragique et dans l'attente d'une transfusion, le remplissage vasculaire doit suivre le principe d'une réanimation à petit volume (low volume fluid resuscitation) qui associe l'introduction précoce des amines pour atteindre les objectifs tensionnels [28]. Lorsque la VCI est dilatée (au moins supérieure à $20 \mathrm{~mm}$ ) sans variation respiratoire, une cause de choc obstructif doit être évoquée. Une fois le pneumothorax suffocant écarté, l'analyse du péricarde recherche une tamponnade péricardique.

\section{Implication opérationnelle de l'échographie dans le triage des blessés et les évacuations sanitaires}

Les avancées technologiques en termes de miniaturisation et de qualité d'image ont permis de positionner les échographes près des équipes médicales dispersées sur les théâtres d'opérations extérieurs. En milieu isolé, son utilisation doit être optimisée [29]. L'impact opérationnel de l'échographe se fait particulièrement ressentir lors du triage en cas d'afflux de blessés et dans la gestion des évacuations sanitaires.

Sur le terrain, l'échographie fournit des éléments parfois discriminants pour déclencher une évacuation sanitaire (exemple : saignement intra-abdominal). Elle devient ensuite essentielle pour la surveillance des lésions, le monitorage hémodynamique et l'ajustement thérapeutique en attendant les vecteurs d'évacuation. En cas de blessés multiples, d'afflux massif ou saturant, l'échographie trouve sa place dans le triage pour la catégorisation des blessés [27-30] en priorisant les patients qui nécessitent un geste d'hémostase urgent. Elle peut ainsi influencer la gestion des flux de patients en modifiant l'ordre d'évacuation sur le terrain, dans les structures chirurgicales et l'ordre de passage au bloc opératoire.

En terrain hostile, les équipes de convoyage se retrouvent à gérer dans leur hélicoptère des patients graves avec un minimum d'informations reposant sur la fiche médicale de l'avant (FMA) et des transmissions orales réalisées rotors 


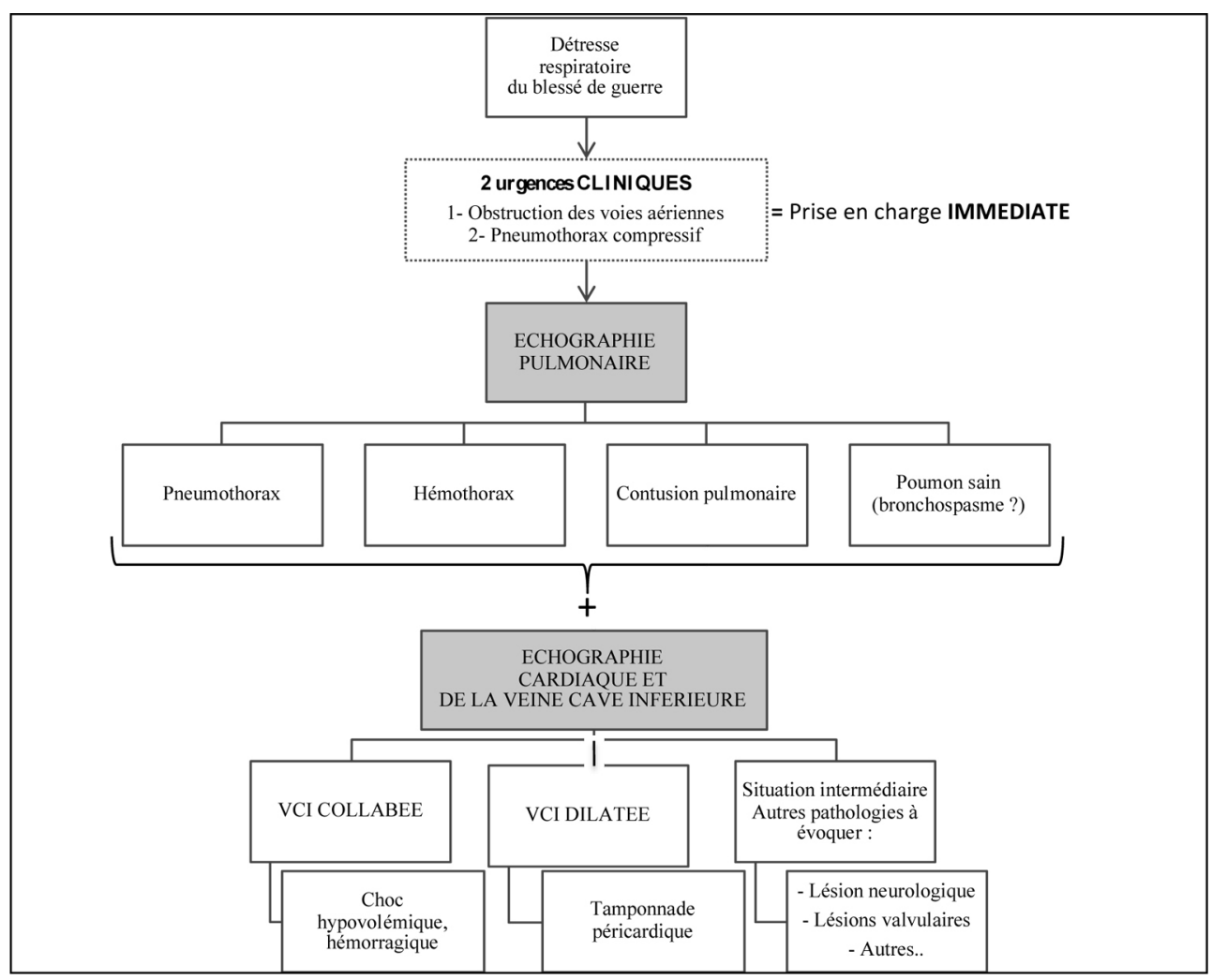

Fig. 5 Diagramme d'aide au diagnostic d'une détresse respiratoire du blessé de guerre avec l'échographie. VCI : veine cave inférieure

tournants. Le contexte sécuritaire incertain ne permet en général pas un posé long du vecteur aérien et une réévaluation commune du patient. La surveillance clinique étant rendue difficile, la figure 5 propose un diagramme d'aide au raisonnement se fondant uniquement sur les données échographiques pour déterminer les causes d'une dégradation respiratoire au cours d'un transport.

\section{Conclusion}

La survie du blessé de guerre dépend de la réalisation de gestes de sauvetage et d'une mise en condition optimale pour l'évacuation sanitaire qui ne doit pas être retardée. L'échographie, outil désormais compatible avec les contraintes de l'avant, peut aider aux prises de décision dès la phase initiale en complément de l'examen clinique pour guider les choix thérapeutiques et les priorités d'évacuation. La pratique de l'échographie est devenue indispensable en médecine de guerre et s'intègre pleinement à la doctrine de la « médicalisation de l'avant ». L'expérience militaire incite à son développement en milieu préhospitalier, que ce soit en métropole ou lors des situations d'exception.

Conflits d'intérêts : les auteurs déclarent ne pas avoir de liens d'intérêts.

\section{Références}

1. École du Val-de-Grâce. Enseignement du sauvetage au combat. Référentiel de formation : 0309/EVDG/DPMO du 30 mars 2012. http://www.ecole-valdegrace.sante.defense.gouv.fr/content/ download/3960/56683/file/Sauvetage \%20au\%20Combat $\% 20$ janvier\%202012.pdf (Dernier accès le 31 janvier 2018)

2. Martinez JY, Wey PF, Letourneur F, et al (2012) Médicalisation de l'avant. Le Congrès SFAR 2012. Médecins. Urgences vitales. $\mathrm{http} / / /$ citerahiadesgenettes.hautetfort.com/media/02/00/ 4074564125.pdf (Dernière accès le 31 janvier 2018)

3. Hornez E, Boddaert G, De Carbonnieres A, et al (2017) Damage control surgery. Le praticien en anesthésie-réanimation 21:148-52

4. Gharahbaghian L, Anderson KL, Lobo V, et al (2017) Pointof-Care ultrasound in austere environments: a complete review of its utilization, pitfalls, and technique for common applications in austere Settings. Emerg Med Clin North Am 35:409-41

5. Duchenne J, Martinez M, Rothmann C, et al (2016) Premier niveau de compétence pour l'échographie clinique en médecine d'urgence. Recommandations de la Société française de médecine d'urgence par consensus formalisé. Ann Fr Med Urgence 6:284-5

6. Rasmussen TE, Baer DG, Cap AP, Lein BC (2015) Ahead of the curve: sustained innovation for future combat casualty care. J Trauma Acute Care Surg 79:61-4

7. Chang R, Eastridge BJ, Holcomb JB. (2017) Remote damage control resuscitation in austere environments. Wilderness Environ Med 28:124-34

8. Sailliol A, Martinaud C, Cap AP, et al (2013) The evolving role of lyophilized plasma in remote damage control resuscitation in the French Armed Forces Health Service. Transfusion 53:65-71

9. Bouzat P, Raux M, David JS, et al (2017) Chest trauma: first 48 hours management. Anaesth Crit Care Pain Med 36:135-145 
10. Eastridge BJ, Mabry RL, Seguin P, et al (2012) Death on the battlefield (2001-2011): implications for the future of combat casualty care. J Trauma Acute Care Surg 73:431-7

11. Keneally R, Szpisjak D (2013) Thoracic trauma in Iraq and Afghanistan. J Trauma Acute Care Surg 74:1292-7

12. Lesquen H, Beranger F, Berbis J, et al (2016) Challenges in warrelated thoracic injury faced by French military surgeons in Afghanistan (2009-2013). Injury 47:1939-44

13. Ivey KM, White CE, Wallum TE, et al (2012) Thoracic injuries in US combat casualties: a 10-year review of Operation Enduring Freedom and Iraqi Freedom. J Trauma Acute Care Surg 73:514-9

14. Aigle L, Castello R, Brevart C, et al (2016) Anterior pulmonary contusion isolated by large-caliber bullet. Mediterra J Emerg Med $24: 30-6$

15. Mabry RL, Edens JW, Pearse L, et al (2010) Fatal airway injuries during Operation Enduring Freedom and Operation Iraqi Freedom. Prehosp Emerg Care 14:272-7

16. Duwat A, Travers S, Deransy R, et al (2017) Cricothyrö̈dotomie par technique SMS (scalpel, mandrin long béquillé, sonde d'intubation) : une alternative à connaître en situation d'exception et d'afflux massif de victimes. Ann Fr Med Urgence 7:319-22

17. Barnard EB, Ervin AT, Mabry RL, et al (2014) Prehospital and en route cricothyrotomy performed in the combat setting: a prospective, multicenter, observational study. J Spec Oper Med 14:35-9

18. Barbe N, Martin P, Pascal J, et al (2014) Repérage de la membrane cricothyrö̈dienne en phase d'apprentissage : valeur ajoutée de l'échographie ? Ann Fr Anesth Reanim 33:163-6

19. Garwe T, Cowan LD, Neas BR, et al (2011) Directness of transport of major trauma patients to a level I trauma center: a propensityadjusted survival analysis of the impact on short-term mortality. J Trauma 70:1118-27

20. Lichtenstein D, Meziere G (2008) Relevance of lung ultrasound in the diagnosis of acute respiratory failure: the BLUE protocol. Chest 134:117-125
21. Volpicelli G, Elbarbary M, Blaivas M, et al (2012) International Liaison Committee on Lung Ultrasound (ILC-LUS) for International Consensus Conference on Lung Ultrasound (ICC-LUS). International evidence-based recommendations for point-of-care lung ultrasound. Intensive Care Med 38:577-91

22. Vignon P, Chastagner C, Berkane V, et al (2005) Quantitative assessment of pleural effusion in critically ill patients by means of ultrasonography. Crit Care Med 33:1757-63

23. Borges JB, Okamoto VN, Matos GF, et al (2006) Reversibility of lung collapse and hypoxemia in early acute respiratory distress syndrome. Am J Respir Crit Care Med 174:268-78

24. Rossaint R, Bouillon B, Cerny V, et al (2016) The European guideline on management of major bleeding and coagulopathy following trauma: fourth edition. Crit Care 20:100

25. Wey PF, Attrait X, Boulanger T, et al (2009) Utilisation pratique de l'échographie pour le triage de blessés de guerre : à propos de la prise en charge de 471 blessés tchadiens en novembre et décembre 2007. Reanoxyo 25:8-11

26. Nagy KK, Lohmann C, Kim DO, Barrett J (1995) Role of echocardiography in the diagnosis of occult penetrating cardiac injury. J Trauma 38:859-62

27. Zhang Z, Xu X, Ye S, Xu L (2014) Ultrasonographic measurement of the respiratory variation in the inferior vena cava diameter is predictive of fluid responsiveness in critically ill patients: systematic review and meta-analysis. Ultrasound Med Biol 40:845-53

28. Butler FK, Holcomb JB, Schreiber MA, et al (2014) Fluid resuscitation for hemorrhagic shock in tactical combat casualty care: TCCC guidelines change. J Spec Oper Med 14:13-38

29. Martin PV, Bila T, Aymart K, et al (2018) Optimization of the ultrasound use in a French role 2 in Mali. Rev Int Serv Sante Forces Armees 91:45-57

30. Wydo SM, Seamon MJ, Melanson SW, et al (2016) Ultrasound in disaster triage: a focused review. Eur J Trauma Emerg Surg 42:151-9 